\title{
Aspectos psicossociais envolvidos na reabilitação de pacientes com cavidade anoftálmica: implicações do uso de prótese ocular
}

\author{
Psychosocial aspects in the rehabilitation of patients with anophthalmic socket: implications \\ of the use of ocular prosthesis
}

Douglas Rangel Goulart ${ }^{1}$, Elizabeth Queiroz ${ }^{2}$, Aline Úrsula Rocha Fernandes ${ }^{3}$, Luciana Martins de Oliveira ${ }^{4}$

\section{RESUMO}

A integridade da face é condição que interfere no cotidiano do indivíduo sendo que a perda do globo ocular tem um impacto psicológico, demandando uma condição de adaptação. Intervenções como a reparação protética parecem produzir mudanças nos âmbitos emocional e comportamental.

Objetivo: Identificar os aspectos psicossociais relacionados à ausência unilateral do globo ocular e avaliar a adaptação dos pacientes usuários de prótese ocular. Métodos: Para realização da pesquisa foi utilizado como instrumento de coleta de dados um roteiro de entrevista adaptado aos objetivos desse trabalho. Este foi composto por duas partes, a primeira referente a dados gerais de identificação do paciente e aspectos socioeconômicos. A segunda parte incluiu dados específicos sobre a perda e reabilitação ocular com 31 questões. Vinte e oito pacientes voluntários foram entrevistados.

Resultados: A maioria era do gênero masculino (53,57\%). A idade variou entre 11 e 67 anos. O trauma foi o fator etiológico prevalente $(60,71 \%)$. Foi encontrada diferença estaticamente significativa $(p<0,05)$ entre os escores dos sentimentos da época da perda e atualmente ( $z$ Wilcoxon=-4,41; $p<0,001$ ).

Conclusão: Os dados evidenciaram dificuldades emocionais no processo inicial de contato com a condição de perda do olho e o papel da prótese como elemento de inclusão social, ressaltando a relevância do trabalho em equipe e do acesso a serviços de protetização para a adaptação desses pacientes.

Descritores: Impacto psicossocial; Enucleação ocular/reabilitação; Enucleação ocular/psicologia; Olho artificial; Anoftalmia/psicologia; Implantes orbitários; Pacientes/ psicologia; Ajustamento social; Recuperação de função fisiológica

\begin{abstract}
Facial integrity is a condition which interferes in the daily life of the individual. The loss of an eyeball has psychological impact, requiring adaptation. Interventions such as prosthetic repair seem to lead to emotional and behavioral change.

Purpose: The objective of this study was to identify the psychosocial aspects related to unilateral anophthalmic socket and evaluate patient adaptation to prosthesis.

Methods: All participants responded to a semi-structured interview. This was composed of two parts, the first regarding the general data of patient identification and socioeconomic aspects. The second part included information about eye loss and rehabilitation, with 31 questions. Twenty-eight volunteers were interviewed.

Results: Most were male (53.57\%). Ages ranged between 11 and 67. Trauma was the prevalent etiological factor $(60.71 \%)$. A significant statistical difference $(p<0.05)$ was found between the scores of the feelings of loss before and after prosthesis ( $z$ Wilcoxon $=-4.41$; $p<0.001$ ).

Conclusions: The data showed emotional difficulties in the initial contact with the loss of an eye and the role of the prosthesis as an element of social inclusion, stressing the importance of teamwork and access to fitting services in adapting these patients.
\end{abstract}

Keywords: Psychosocial impact; Eye enucleation/rehabilitation; Eye enucleation/psychology; Eye, artificial; Anophthalmos/psychology; Orbitalimplants; Patients/psychology; Social adjustment; Recovery of function

\section{INTRODUÇÃO}

A face humana permite ao homem exprimir seus sentimentos e se comunicar ${ }^{(1)}$. Pessoas com deformidades faciais podem ter dificuldades em estabelecer vínculos afetivos, em organizar a vida diante das novas circunstâncias, além de apresentar sentimentos de inferioridade e rejeição em relação ao meio de convivência ${ }^{(2)}$

A perda do globo ocular compromete a normalidade facial e em função do impacto psicológico provocado pode conduzir o individuo a um grau de desestruturação temporária ou permanente. Assim a reabilitação da face através de cirurgias e/ou próteses representa um importante instrumento de adaptação, sendo fun- damental a reparação estética. Há também o caráter funcional e de proteção da cavidade anoftálmica ${ }^{(3)}$.

Na análise das diferentes definições de reabilitação é ressaltado seu caráter subjetivo e processual, ou seja, uma relação de causaefeito que pode gerar demandas específicas ao longo do tempo, portanto sujeita a uma variação dependendo de características, tais como patologia, idade, rede de apoio, recursos pessoais, acesso a serviços de saúde, entre outras. Além disso, o trabalho em equipe tem sido reconhecido como um fator importante na qualidade dos serviços prestados por distintas categorias profissionais e diferentes tipos de instituições ${ }^{(4)}$.
Submitted for publication: June 17, 2011

Accepted for publication: October 10, 2011

Study carried out at the Universidade de Brasília - UniB.

Student, Faculdade de Odontologia, Universidade de Brasília - UnB - Brasília (DF), Brazil.

2 Psychologist, Departamento de Psicologia Escolar e do Desenvolvimento, Instituto de Psicologia, Brasília (DF), Brazil.

Dentist, Associate professor, Departamento de Odontologia, Faculdade de Ciências da Saúde, Universidade de Brasília - UnB - Brasília (DF), Brazil.

${ }^{4}$ Student, Instituto de Psicologia, Universidade de Brasília - UnB - Brasília (DF), Brazil.

Projeto de pesquisa aprovado pelo Comitê de Ética em Pesquisa da Faculdade de Ciências da Saúde da Universidade de Brasília sob o no 018/10.
Funding: No specific financial support was available for this study.

Disclosure of potential conflicts of interest: D.R.Goulart, None; E.Queiroz, None; A.U.R.Fernandes, None; L.M.de Oliveira, None.

Correspondence address: Douglas Rangel Goulart. Departamento de Odontologia - Universidade de Brasília - Campus Universitário Darcy Ribeiro - Brasília, Distrito Federal - 70910-900 - Brazil E-mail: douglasrgoulart@gmail.com 
A prótese bucomaxilofacial tem por objetivos básicos restaurar a estética e proteger a área danificada, além de promover uma reestruturação psicológica até certo ponto, podendo levar à resolução de pequenos transtornos, porém para grandes traumas psíquicos 0 acompanhamento psicológico individualizado é indispensável(5-7).

Nesse contexto, é imprescindível que o cirurgião dentista especialista em prótese bucomaxilofacial, tenha conhecimento dos aspectos psicológicos, visto que a prótese pode não significar reabilitação ${ }^{(1,2)}$. Mais do que isso, tal estratégia pode funcionar como um agente de depressão para o paciente, se não corresponder ao seu desejo inconsciente ou consciente, de ter de volta a parte perdida da face ${ }^{(3)}$. O tratamento, portanto, abrange além dos procedimentos técnicos, os aspectos psicossociais que circundam o contexto em que a perda ocular ocorre, possibilitando aos pacientes melhores condições de convívio com a deficiência e suas consequências ${ }^{(7)}$.

Para promover um atendimento em reabilitação mais abrangente, aliando o conhecimento técnico-científico em prótese ao psicológico, o presente estudo teve por objetivo identificar os aspectos psicossociais relacionados à ausência unilateral do globo ocular e avaliar a adaptação dos pacientes usuários de prótese ocular.

\section{MÉTODOS}

Foi realizado um estudo transversal com pacientes usuários de prótese ocular unilateral no período de abril a outubro de 2010. 0 estudo seguiu as recomendações de pesquisa envolvendo seres humanos, adotadas pela Resolução 196/96, do Conselho Nacional de Saúde tendo sido aprovado pelo Comitê de Ética em Pesquisa da Faculdade de Ciências da Saúde da Universidade de Brasília sob o nº 018/10. Para realização da pesquisa foi utilizado como instrumento de coleta de dados um roteiro de entrevista elaborado a partir de estudos similares ${ }^{(7,8)}$, adaptado aos objetivos desse trabalho.

O instrumento de coleta de dados foi composto por duas partes, a primeira referente a dados gerais de identificação do paciente e aspectos socioeconômicos. A segunda parte incluiu dados específicos sobre a perda e reabilitação ocular com 31 questões (objetivas e subjetivas), que contemplam informações sobre a perda ocular e o processo de reabilitação.

A composição da amostra foi precedida de uma revisão dos prontuários dos pacientes atendidos pelo serviço de prótese bucomaxilofacial desde 2004. Foram identificados 63 prontuários de pacientes com ausência do globo ocular. Os contatos telefônicos dos pacientes foram registrados e diferentes tentativas de recrutamento foram realizadas. Os pacientes que se dispuseram a participar da pesquisa preencheram um Termo de Consentimento Livre e Esclarecido. Para os pacientes menores de 18 anos os responsáveis legais, assinaram o Termo e participaram como fonte adicional de informação na entrevista.

Os dados quantitativos foram avaliados através de estatísticas descritivas e análises correlacionais com auxílio do pacote estatístico Statistical Package for Social Sciences (SPSS) para Windows, versão 18.0. As respostas às questões subjetivas foram submetidas à análise qualitativa por eixos temáticos.

\section{RESULTADOS}

A amostra foi composta por 28 pacientes voluntários, o que correspondeu a $44,44 \%$ do total de pacientes identificados. Vinte e sete pacientes não foram localizados porque o número do telefone não estava válido; quatro não estavam usando a prótese ocular; dois não compareceram quando marcados, um paciente faleceu no período e uma paciente tinha apenas cinco anos de idade e a equipe optou por não inclui-la em função das especificidades da idade. A tabela 1 apresenta a caracterização da amostra:

A amostra foi constituída de 53,57\% do gênero masculino. A idade variou entre 11 e 67 anos. Quanto à escolaridade 67,85\% da amostra não havia concluído o Ensino Médio. A maioria dos partici-

\section{Tabela 1. Dados sociodemográficos da população estudada relacionados à cavidade anoftálmica e adaptação de prótese ocular}

\begin{tabular}{crlr}
\hline $\begin{array}{c}\text { Gênero } \\
\text { Feminino }\end{array}$ & $46,43 \%$ & $\begin{array}{l}\text { Escolaridade } \\
\text { Analfabeto(a) }\end{array}$ & $7,14 \%$ \\
$\begin{array}{c}\text { Masculino } \\
\text { Idade }\end{array}$ & $53,57 \%$ & $\begin{array}{l}\text { Ensino fundamental incompleto } \\
\text { 10 a } 15\end{array}$ & $35,71 \%$ \\
16 a 20 & $7,14 \%$ & Ensino fundamental completo & $7,14 \%$ \\
21 a 25 & $3,14 \%$ & Ensino médio incompleto & $17,86 \%$ \\
26 a 30 & $10,71 \%$ & Ensino superior completo & $28,57 \%$ \\
31 a 35 & $3,57 \%$ & & $0,00 \%$ \\
36 a 40 & $10,71 \%$ & Renda familiar* & $3,57 \%$ \\
41 a 45 & $3,57 \%$ & Entre 1 a 2 salários \\
46 a 50 & $14,29 \%$ & Entre 2 e 3 salários & \\
51 a 55 & $7,14 \%$ & Entre 3 e 4 salários & $35,71 \%$ \\
56 a 60 & $7,14 \%$ & Entre 4 e 5 salários & $25,00 \%$ \\
61 a 65 & $10,71 \%$ & Acima de 5 salários & $32,14 \%$ \\
66 a 70 & $14,29 \%$ & & $3,57 \%$ \\
\hline
\end{tabular}

* = com base no salário mínimo nacional vigente na época da entrevista $\mathrm{R} \$ 510,00$

pantes (92,86\%) declarou seguir uma religião, principalmente a Católica Apostólica Romana (53,57\%), seguida da Protestante (28,57\%), do Espiritismo (7,14\%) e Testemunhas de Jeová (3,57\%). Com relação à ocupação, 46,43\% da amostra declarou estar inserida no mercado de trabalho, 28,57\% eram aposentados, 10,71\% estudantes, $7,14 \%$ desempregados e 7,14\% desempenhavam o papel de dona de casa. As informações sobre renda foram colhidas com base no salário mínimo nacional vigente na época da entrevista ( $\mathrm{R} \$ 510,00)$, para mais da metade da amostra $(60,71 \%)$ renda familiar variou entre um e três salários mínimos. O estado civil predominante foi o solteiro $(35,71 \%)$, seguido de casado $(32,14 \%)$, viúvo $(17,86 \%)$ e divorciado $(14,29 \%)$.

A tabela 2 apresenta os dados relacionados à história da perda do globo ocular e da reparação protética. O trauma foi o fator etiológico prevalente (60,71\%), seguido do glaucoma (10,71\%), anoftalmia congênita (7,14\%), neoplasia maligna (7,14\%), sarampo (7,14\%), diabetes mellitus (3,57\%) e causa não identificada (3,57\%). A ausência do globo ocular foi identificada mais frequentemente no lado esquerdo da face $(71,43 \%)$. A maioria dos pacientes $(82,14 \%)$ realizou um ou mais procedimentos cirúrgicos no globo ocular. A maior parte dos sujeitos da pesquisa $(89,29 \%)$ relatou ter recebido informações sobre a possibilidade de perda ocular, sendo que $64,29 \%$ foram informados no momento da cirurgia sobre a necessidade de usar uma prótese. Para 35,71\% a prótese foi adaptada depois de mais de cinco anos da perda. A reparação protética foi realizada principalmente em instituições públicas (67,86\%).

A tabela 3 apresenta os dados quantitativos relacionados às implicações psicossociais e à prótese. A maior parte da população estudada recebeu apoio da família (89,29\%). Apenas cinco pacientes receberam acompanhamento psicológico após a perda do globo ocular, sete pacientes consideraram esse acompanhamento um importante auxílio para a adaptação de pessoas com anoftalmia congênita ou que perderam o globo ocular. Metade da amostra não considerou a prótese como instrumento de modificação do seu relacionamento interpessoal, sendo que 46,43\% observaram modificações positivas. Para $32,14 \%$ a perda ocular repercutiu de forma negativa na rotina de vida. Quanto à religiosidade o fato de ter perdido ou não ter o globo ocular não alterou a religiosidade de 89,29\% dos participantes, dois participantes passaram e ter maior crença e apenas um mudou de religião.

A maior parte da amostra $(53,57 \%)$ relatou não ter qualquer limitação relacionada à ausência do globo ocular e/ou ao uso de prótese, para aqueles que identificaram, as limitações relatadas foram: tra- 
Tabela 2. História da perda do globo ocular e indicação de reabilitação protética

\begin{tabular}{lrlr}
\hline Etiologia da perda ocular & & Recebeu informação sobre a necessidade de usar uma prótese ocular \\
Trauma & $60,71 \%$ & Sim, pelo médico antes de realizar uma cirurgia & Sim, por outras pessoas após ter removido o globo ocular \\
Glaucoma & $10,71 \%$ & Sim, pelo médico em uma consulta de acompanhamento & $14,28 \%$ \\
Neoplasia maligna & $7,14 \%$ & Não, procurou se informar por iniciativa própria & $10,71 \%$ \\
Sarampo & $7,14 \%$ & Momento da reabilitação protética & $10,71 \%$ \\
Anoftalmia congênita & $7,14 \%$ & No mesmo ano da perda ocular & $32,14 \%$ \\
Diabetes mellitus & $3,57 \%$ & 1 ano após a perda ocular & $14,28 \%$ \\
Não identificada & $3,57 \%$ & 2 anos após a perda ocular & $14,28 \%$ \\
Local em que foi realizada & & 3 anos após a perda ocular & $3,57 \%$ \\
a reabilitação protética & & 4 anos após a perda ocular & $3,57 \%$ \\
Hospital universitário & $64,29 \%$ & 5 anos após a perda ocular & $7,14 \%$ \\
Clínica particular & $32,14 \%$ & Mais de 5 anos após a perda ocular \\
Outro local público & $3,57 \%$ & $35,71 \%$ \\
\hline
\end{tabular}

Tabela 3. Aspectos psicossociais relacionados à prótese ocular na população portadora de cavidade anoftálmica

\begin{tabular}{|c|c|c|c|}
\hline Informações sobre a rede de apoio & & Alteração da rotina de vida após a perda ocular & \\
\hline Recebeu apoio da família & $89,29 \%$ & Não alterou a rotina & $64,29 \%$ \\
\hline Relatou sentir falta do apoio da família & $14,28 \%$ & Alterou a rotina de forma negativa & $32,14 \%$ \\
\hline Fez acompanhamento psicológico & $17,86 \%$ & Alterou a rotina de forma positiva & $3,57 \%$ \\
\hline $\begin{array}{l}\text { Alteração da religiosidade } \\
\text { após a perda ocular }\end{array}$ & & $\begin{array}{l}\text { Alteração no relacionamento interpessoal após } \\
\text { a reabilitação com a prótese ocular }\end{array}$ & \\
\hline Mudou de religião & $3,57 \%$ & Alterou de forma negativa & $3,57 \%$ \\
\hline Passou a ter maior crença & $7,14 \%$ & Alterou de forma positiva & $46,43 \%$ \\
\hline Não alterou & $89,29 \%$ & Não alterou & $50,00 \%$ \\
\hline
\end{tabular}

balhar (7,14\%); estudar (7,14\%); costurar (7,14\%); usar maquiagem (7,14\%); obter Carteira Nacional de Habilitação destinada à condução de veículos de transporte de passageiros (acima de oito pessoas) e de veículos para transporte de carga acima de $3.500 \mathrm{~kg}$ (7,14\%); nadar (7,14\%); e olhar filmes com imagens codificadas de forma a dar ao espectador a ilusão de uma terceira dimensão espacial (3,57\%).

A análise qualitativa foi dividida em quatro eixos temáticos: percepções relacionadas à prótese; expectativas quanto ao uso da prótese; autodescrição utilizando a reabilitação protética como referencial temporal e; sentimentos relacionados temporalmente da perda à reabilitação protética.

As percepções relacionadas à prótese foram categorizadas em positivas ou indiferentes, sendo a prótese classificada quanto à funcionalidade e presença de sintomas. As atribuições positivas (ótima, excelente, muito boa e boa), foram prevalentes $(85,71 \%)$, ainda que $16,66 \%$ desses participantes apresentassem algum sintoma relacionado à prótese, principalmente secreção e dor. Nesse grupo que valorizava a prótese, um participante considerou que ela não cumpria com a funcionalidade estética. A atribuição indiferente foi relatada por quatro participantes da amostra, sendo que para dois desses a prótese não cumpria a funcionalidade estética e três relataram presença de sintomas.

A estética foi a principal expectativa relacionada à prótese (71,42\%). Também foram identificadas a perspectiva irreal de voltar a enxergar (14,28\%), expectativas com foco na dificuldade de adaptação $(10,71 \%)$ e nenhuma expectativa $(3,57 \%)$.

Na autodescrição anterior ao uso da prótese, 32,14\% da amostra relataram timidez, 25\% baixa autoestima, 14,28\% preocupação em esconder a diferença, e 10,71\% ser alvo de curiosidade. Para 21,42\% da amostra, a prótese não funcionou como elemento de mudança de atitudes, comportamentos e sentimentos. Alguns participantes descreveram situações de mudança sem estabelecer a relação entre a cavidade anoftálmica e a reabilitação protética (10,71\%). Após a prótese, os eixos temáticos representaram o tipo de mudança que os participantes associam com o período atual, pós-reabilitação. Desta- caram-se mudança positiva no relacionamento interpessoal (25\%), perspectivas de que a situação atual é melhor (25\%), percepção de não ser mais foco de curiosidade (14,28\%), valorização da prótese $(10,71 \%)$, melhora na autoestima $(7,14 \%)$ e associação a um estado de normalidade (7,14\%).

Para avaliar os sentimentos temporalmente da época da perda à situação atual, foram utilizadas três questões: uma subjetiva e duas objetivas. A questão subjetiva antecedeu as objetivas de forma a não induzir respostas. As questões objetivas abordaram os principais sentimentos encontrados na literatura relacionados à perda de parte da face ${ }^{(1,2,4,7)}$ (tristeza, vergonha, timidez, medo, inferioridade, insegurança, preocupação em esconder, raiva e culpa). Para cada sentimento os indivíduos indicaram um grau (nenhum, pouco, muito e demais) em dois momentos: na época da perda e atualmente.

Os sentimentos associados espontaneamente ao momento da perda foram tristeza $(57,14 \%)$, incapacidade $(14,28 \%)$, vergonha $(10,71 \%)$, depressão (10,71\%), revolta $(7,14 \%)$ e medo (3,57\%). Parte da amostra relatou não recordar os sentimentos vivenciados (14,28\%). Quando questionados sobre os sentimentos atuais, 42,85\% da amostra relatou respostas positivas (bem, ótimo e feliz), 17,86\% relataram perspectiva de melhora. Uma parte da amostra relatou sentimentos associados a um processo de ajustamento não concluído, evidenciado por respostas com conformidade $(7,14 \%)$, incapacidade $(3,57 \%)$ e desajustamento (3,57\%). Sete participantes associaram os sentimentos atuais com "normalidade" (25\%).

Nas questões objetivas os sentimentos listados apresentaram uma diminuição em número de relatos e grau da época da perda para o momento atual. A tristeza foi relatada na época da perda por $28,57 \%$ da amostra com grau demais, $17,86 \%$ muito, 32,14\% pouco e $21,43 \%$ nenhum. No momento atual prevaleceram as respostas nenhum (75\%) e pouco $(21,43 \%)$.

A inferioridade apresentou redução significativa de intensidade, da época da perda (39,29\% nenhum, 25\% pouco, 17,86\% muito e $17,86 \%$ demais) para o momento atual (78,57\% nenhum e $21,43 \%$ pouco). A timidez foi relatada na época da perda de forma igual para todos os graus (25\%), no momento atual prevaleceu nenhum 
(71,43\%), seguido de pouco $(21,43 \%)$ e muito (7,14\%). Quanto à insegurança na época da perda, 39,29\% da amostra relatou o grau nenhum, $25 \%$ pouco, $17,86 \%$ muito e $17,86 \%$ demais. No momento atual foram indicados apenas os graus nenhum (78,57\%) e pouco $(21,43 \%)$. O sentimento vergonha foi relatado na época da perda com graus nenhum $(32,14 \%)$, pouco $(21,43 \%)$, muito $(28,57 \%)$ e demais (17,86\%), e no momento atual apresentou predominância de nenhum (64,29\%), seguido de pouco $(32,14 \%)$ e muito $(3,57 \%)$.

Para realizar a análise estatística correlacional os graus de sentimentos apontados pelos indivíduos nas questões objetivas foram convertidos em escores. Para cada um dos nove sentimentos questionados foi atribuído um número de acordo com o grau indicado (0-nenhum, 1-pouco, 2-muito e 3-demais). Um escore total foi gerado a partir da soma dos escores atribuídos para cada um dos dois momentos: na época da perda e atualmente.

Foi encontrada diferença estatisticamente significativa $(p<0,05)$ entre os escores dos sentimentos da época da perda e atualmente ( $z$ Wilcoxon=-4, 41; $p<0,001$ ), sendo que para o grupo feminino esta diferença é levemente mais forte (Masculino, $z$ Wilcoxon=-3,08; $p=0,002 ; r=-0,79$; Feminino, $z$ Wilcoxon=-3,18; $p=0,001 ; r=-0,88$ ).

O tempo decorrido da perda à reparação protética teve correlação positiva, moderada e estatisticamente significativa quando relacionado aos escores dos sentimentos na época da perda ocular (rho=-0,34; $p=0,04)$ e atualmente ( $r$ o $=-0,44 ; p=0,01)$.

Os escores dos sentimentos da época da perda obtiveram correlação negativa, moderada e estatisticamente significativa com o número de cirurgias realizadas ( $r h o=-0,33 ; p=0,05)$. Entretanto não foi encontrada correlação estatisticamente significativa entre o número de cirurgias e os escores dos sentimentos atuais (rho=-0,20; $p=0,15$ ).

Não foi encontrada correlação estatisticamente significativa quando os escores dos sentimentos da época da perda foram relacionados ao gênero ( $U$ Mann-Whitney=72,50; $p=0,25 ; r=0,22$ ).

A idade cronológica relatada no momento da entrevista teve correlação negativa, moderada e estatisticamente significativa com os escores dos sentimentos atuais ( $r h o=-0,37 ; p=0,03$ ).

Os escores dos sentimentos atualmente não apresentaram correlação estatisticamente significativa quando relacionados à presença de sintomas oculares ligados à prótese ( $U$ Mann-Whitney=68; $p=0,41, r=0,17)$ e com a funcionalidade estética da prótese (U MannWhitney=36; $p=0,94, r=0,02$ ).

$\mathrm{Na}$ análise dos sintomas oculares relacionados à prótese, não foram encontradas diferenças entre o grupo que apresenta sintomatologia daquele que não apresenta no que se refere ao número de próteses que já usou ( $U$ Mann-Whitney=72; $p=0,53, r=0,13$ ). Além disso, não foram encontradas diferenças na frequência da sintomatologia entre as pessoas que realizaram o tratamento em local público daquelas que realizaram em clínicas privadas $\left(X^{2}(1)=0,58 ; p=0,44\right)$.

Após a realização das entrevistas os pacientes foram avaliados clinicamente, para oito desses as próteses foram substituídas por apresentarem condições estéticas e funcionais insatisfatórias. Fizeram parte desse grupo dois pacientes dos três que não consideravam a prótese como funcional do ponto de vista estético.

Os escores dos sentimentos atualmente não apresentaram diferenças estatisticamente significativas entre os grupos que necessitavam ou não da substituição da prótese ocular (U Mann-Whitney=61,5; $p=0,33, r=0,18)$.

A mediana das idades dos pacientes com necessidade de substituir a prótese ocular foi de 31,5 anos, para o grupo de pacientes que não necessitavam da substituição foi de 51 anos. Entretanto não foi encontrada diferença estatisticamente significativa entre esses grupos ( $U$ Mann-Whitney=50; $p=0,13, r=0,18$ ).

\section{DISCUSSÃO}

Nesta pesquisa foi encontrada diversidade de religião, estado civil e ocupação. Em comum observou-se baixa renda familiar e limitada escolaridade, que têm sido associadas à população que necessita deste tipo de serviço especializado ${ }^{(5,9)}$.

Das mutilações faciais, a região ocular tem sido descrita como a mais atingida ${ }^{(10)}$, e o trauma como fator etiológico prevalente ${ }^{(11,12)}$, dado corroborado por resultados deste trabalho.

Houve atrasos na reparação protética, apesar da maior parte da amostra ter recebido informações sobre a necessidade de usar uma prótese ocular na época da realização da cirurgia. Esse fato deve-se a escassez de centros especializados para tratamento das mutilações faciais ${ }^{(1,3,5)}$. Como decorrência, os pacientes têm dificuldade de acesso, ficam excluídos do convívio social e familiar, carregando uma carga emocional que implica na perda de qualidade de vida ${ }^{(13)}$. Isso é especialmente sério visto que a noção de gravidade de uma deficiência é subjetiva uma vez que sofre influência de variáveis do contexto de vida do paciente, principalmente o acesso a serviços de saúde e recursos econômicos. Não se pode ignorar que a impossibilidade de aquisição de uma prótese, por exemplo, pode aumentar o impacto provocado pela perda do globo ocular à vida de uma pessoa.

O apoio social é frequentemente descrito como tendo um importante efeito positivo no ajustamento psicológico após uma deficiência e, por essa razão, a proposta de intervenção também deve integrar a família. A atenção aos familiares nos processos de avaliação e programas de reabilitação destacam os recursos do paciente que podem contribuir ou dificultar seu processo de inserção ou reinserção social. Assim, a família deve ser considerada dentro do contexto da deficiência, pois esta não atinge apenas o sujeito, mas também a todos os seus membros ${ }^{(7)}$. No estudo a maior parte da amostra (89,29\%) recebeu apoio da família.

Estudos evidenciam que o suporte para as pessoas com algum tipo de deformidade facial é dado principalmente pela família e pelo parceiro ${ }^{(13)}$. O suporte social parece ser efetivo quando se aproxima das necessidades do indivíduo, e deve ser considerado pelos profissionais de saúde no planejamento da reabilitação(14).

Chama a atenção o fato de 89,29\% da amostra responder que a perda ocular não alterou sua religiosidade. Estudos têm mostrado a importância da religiosidade como uma estratégia de enfrentamento que favorece o lidar com situações de perda ${ }^{(15)}$. Uma possibilidade é que os pacientes, por terem a visão garantida pelo olho não afetado não tenham que lidar com o aspecto funcional decorrente. Tal hipótese é corroborada pelo dado de que a única paciente que relatou mudança de religião é a que é cega.

Apesar do pequeno número de pacientes (4) que não atribuíram características positivas à prótese ocular, a maior parte destes apresentou déficit estético (2) e relataram presença de sintomas (3). No entanto o fato de a prótese estar clinicamente insatisfatória não modificou a percepção positiva desta por seis pacientes. Um maior número de próteses utilizadas e/ou ser reabilitado em uma instituição pública ou privada não assegura a ausência de sintomas.

A estética foi a principal expectativa relacionada à prótese. Também foram identificadas a perspectiva irreal de voltar a enxergar e expectativas com foco na dificuldade de adaptação. A análise dessas respostas ressalta a importância da comunicação que deve ser estabelecida entre os profissionais de saúde e o usuário(16).

A comparação da autodescrição antes e depois do uso da prótese permite identificar a importância da variável tempo em reabilitação, visto que as demandas decorrentes da perda de um globo ocular podem associar-se a questões cotidianas que pressupõem adaptação. É evidente que as pessoas têm necessidades específicas ao longo de sua vida, considerando as diferentes etapas de desenvolvimento.

Os participantes evidenciaram mudanças positivas em relação aos sentimentos experienciados, cabe à consideração de que o evento em evidência é a reabilitação e não a perda, o que pode direcionar o foco de análise dos participantes. Ainda é preciso considerar que o tempo decorrido desde a perda faz com que as lembranças possam 
ser minimizadas. Além disso, é sabido que lidar com uma condição adversa de desenvolvimento representa a possibilidade de ampliação de recursos adaptativos que favorecem percepções positivas diante do que poderia ter sido.

Soma-se a isso a noção de normalidade trazida por alguns participantes. Tradicionalmente, a noção de normalidade aparece associada à questão da diferença, realçada pelo preconceito decorrente da variação existente. A introdução da noção do estigma explorou as implicações desse conceito para as práticas sociais ${ }^{(17)}$.

Os pacientes mais velhos relataram menos sentimentos associados à perda do globo ocular. Tal resposta pode ser atribuída à resiliência, capacidade que o indivíduo possui para superar adversidades e sair fortalecido(18).

As investigações sobre os fatores psicossociais nas diferentes enfermidades ganham cada vez mais importância no tratamento e na reabilitação ${ }^{(16,19)}$. Porém, estudos específicos sobre o impacto da perda de parte da face e o papel da reabilitação protética na região facial são escassos ${ }^{(13)}$.

\section{CONCLUSÃO}

Foi constatada uma perda relevante de dados cadastrais dos pacientes em pouco tempo de funcionamento do serviço de prótese ocular na instituição pesquisada, o que se deve em parte pela falta de um mecanismo de atualização de dados do prontuário, e ao fato de que muitos pacientes não têm acesso ao serviço de telefonia fixa, dispondo apenas de aparelhos móveis ou de contatos vinculados aos locais de trabalho, com mudanças frequentes.

Observa-se que ainda que não tenham sido beneficiados por um acompanhamento psicológico específico, os pacientes estudados apontaram a prótese como o mecanismo compensatório para adaptação a sua condição. Sabe-se que as atitudes dos profissionais influenciam de forma direta a maneira como o paciente se percebe após uma lesão e como incorpora esta nova condição. Na falta de uma política mais ampla de atenção ao paciente com ausência do globo ocular, o desenvolvimento de procedimentos adequados de orientação pode ajudar o paciente a não ter expectativas irrealistas em relação ao tratamento, o que pode resultar em melhores relações interpessoais. Em outras palavras, a atuação de cada profissional pode contribuir para o resultado final dos programas de reabilitação, sendo o treinamento e constituição de equipes imprescindíveis para a qualificação dos atendimentos.

\section{REFERÊNCIAS}

1. Cardoso MS, Araújo PG, Cardoso AJ, Cardoso, SM, Morais, LC. Implicações psicossociais em pacientes com perda do globo ocular. Rev Cir Traumatol Buco-maxilo-fac. 2007;7(1):79-84

2. Amaro TA, Belfort R, Erwenne CM. Estudo psicológico de pacientes enucleados por trauma ou tumor ocular em uso de prótese. Acta Oncol Bras. 2000;20(4):138-42.

3. Loretto NR, Cardoso MS, Cardoso SM, Cardoso AJ, Morais LC. Importância da reabilitação protética da região óculo-palpebral: relato de caso. Odontol Clín-cient. 2008;7(2):151-5.

4. QueirozE. Trabalho em equipe no contexto hospitalar: uma investigação sobre os aspectos comunicacionais envolvidos na tomada de decisão clínica em instituição de reabilitação [Tese]. Brasília: Instituto de Psicologia, Universidade de Brasília; 2003.

5. Cabral LG, Martelli Júnior H, Leite DM, Sabatini Júnior D, Freitas AB, Miranda RT, et al. Perfil biopsicossocial de portadores de anoftalmia no sul de Minas Gerais - Brasil. Arq Bras Oftalmol. 2008;71(6):855-9

6. Silveira M, Silveira ME. Psicologia e reabilitação: a importância da reabilitação facial para o paciente e sua subjetividade. Psicópio: Rev Virtual Psicol Hosp Saúde. 2005:1(2):13-7.

7. Botelho NL, Volpini M, Moura EM. Aspectos psicológicos em usuários de prótese ocular. Arq Bras Oftalmol. 2003;66(5):637-46.

8. Nicodemo D, Ferreira LM. Formulário do perfil psicossocial do paciente anoftálmico com indicação de prótese ocular. Arq Bras Oftalmol. 2006;69(4):463-70.

9. Ahn JM, Lee SY, Yoon JS. Health-related quality of life and emotional status of anophthalmic patients in Korea. Am J Ophthalmol. 2010;149(6):1005-11.

10. Araújo Filho RC, Cardoso MS, Cardoso AJ, Pereira JR, Souza EH, Macedo CB. Fatores etiológicos das mutilações buco-maxilo-faciais em pacientes atendidos no serviço de prótese buco da FOP/UPE. Odontol Clín-cient. 2006;5(3):203-6.

11. Goiato MC, Mancuso DN, Fernandes AUR, Dekon SFC. Estudo sobre as causas mais frequentes de perdas oculares. Arq Odontol. 2004;40(3):207-86.

12. Saeed MU, Chang BY, Khandwala M, Shivane AG, Chakrabarty A. Twenty year review of histopathological findings in enucleated/eviscerated eyes. J Clin Pathol. 2006;59(2):153-5.

13. Rumsey N, Harcourt D. Body image and disfigurement: issues and interventions. Body Image. 2004;1(1):83-97.

14. Clarke A. Psychosocial aspects of facial disfigurement: problems, management and the role of a lay-led organization. Psychol Health Med. 1999;4(2):127-42.

15. Faria JB, Seidl EM. Religiosidade e enfrentamento em contextos de saúde e doença: revisão da literatura. Psicol Reflex Crit. 2005;18(3):381-9.

16. Straub RO. Psicologia da saúde. Porto Alegre: Artmed; 2005.

17. Goffman E. Estigma-notas sobre a manipulação da identidade deteriorada. Rio de Janeiro: Editora Guanabara; 1988.

18. Rudnicki T. Resiliência e o trabalho do psicólogo hospitalar: considerações iniciais. Rev SBPH [Internet] 2007 [cited 2010 Jun 27];10(2):83-92. Available from: http://pepsic.bvsalud. org/pdf/rsbph/v10n2/v10n2a10.pdf

19. Sarafino EP. Health psychology: biopsychosocial interactions. $6^{\text {th }}$ ed. New Jersey: John Wiley \&Sons; 2006 